\title{
Chemotherapy resistance and oncogene expression in non-small cell lung cancer
}

Thomas A. d'Amato, MD, PhD, ${ }^{a}$ Rodney J. Landreneau, MD, ${ }^{b}$ William Ricketts, PhD, ${ }^{c}$ Weidong Huang, MD, PhD, ${ }^{c}$ Ricardo Parker, $\mathrm{PhD},{ }^{\mathrm{c}}$ Eugene Mechetner, $\mathrm{MD}^{\mathrm{c}}{ }^{\mathrm{I}}$ Ing-Ru $\mathrm{Yu}, \mathrm{MS}^{\mathrm{c}}$ and James D. Luketich, $\mathrm{MD}^{\mathrm{b}}$

Objectives: Empiric chemotherapy for patients with non-small cell lung cancer who have undergone resection is recommended without knowledge of the tumor's specific biologic characteristics, and many patients may not benefit. In vitro chemotherapy resistance is associated with clinical unresponsiveness in some tumors, and in lung cancer, chemotherapy resistance is prevalent. Multiple-agent chemotherapy resistance and association of chemotherapy resistance with molecular markers are described.

Methods: Chemotherapy resistance to doublets-carboplatin and paclitaxel, cisplatin and navelbline, cisplatin and docetaxel, and cisplatin and gemcitabine-was analyzed in 4571 non-small cell lung cancer tumors with the extreme drug resistance assay. Chemotherapy resistance is defined as follows: extreme drug resistance, $1 \mathrm{SD}$ above the median chemotherapy resistance; intermediate drug resistance, between the median and extreme drug resistances; and low drug resistance, $1 \mathrm{SD}$ below the median. Chemotherapy resistance was compared with DNA ploidy measured by flow cytometry, and markers p53 and epithelial growth factor receptor were assayed by immunohistochemistry.

Results: Tumors with extreme or intermediate drug resistance were noted in $30 \%$ to carboplatin-paclitaxel, in $24 \%$ to cisplatin-navelbline, in $42 \%$ to cisplatingemcitabine, and in $27 \%$ to cisplatin-docetaxel. Extreme or intermediate drug resistance to at least one drug occurred in $74 \%$ to carboplatin-paclitaxel, in $68 \%$ to cisplatin-navelbline, in $88 \%$ to cisplatin-gemcitabine, and in $68 \%$ to cisplatindocetaxel. More intermediate plus extreme chemotherapy resistances occurred in aneuploid tumors to etoposide (53\% vs $36 \%, P=.0002)$ and topotecan (48\% vs

From Jefferson Medical College, Division of Cardiothoracic Surgery, Philadelphia, $\mathrm{Pa}^{\mathrm{a}}$; the University of Pittsburgh Medical Center Presbyterian-Shadyside, Heart, Lung and Esophageal Surgery Institute, Pittsburgh, $\mathrm{Pa}^{\mathrm{b}}$; and Oncotech, Inc, Tustin, Calif.

Read at the Thirty-second Annual Meeting of the Western Thoracic Surgical Association, Sun Valley, Idaho, June 21-24, 2006.

Received for publication June 19, 2006; revisions received Sept 25, 2006; accepted for publication Oct 9, 2006.

Address for reprints: Thomas A. d'Amato, $\mathrm{MD}, \mathrm{PhD}$, Division of Cardiothoracic Surgery, Jefferson Medical College of Thomas Jefferson University, 1025 Walnut St, Suite 607, Philadelphia, PA 19107 (E-mail: thomas.damato@jefferson.edu).

J Thorac Cardiovasc Surg 2007;133:352-63 $0022-5223 / \$ 32.00$

Copyright $\odot 2007$ by The American Association for Thoracic Surgery

doi:10.1016/j.jtcvs.2006.10.019 $36 \%, P=.0094)$, with less intermediate or extreme chemotherapy resistance to gemcitabine ( $88 \%$ vs $81 \%, P=.0345)$. p53-Positive tumors had more intermediate or extreme resistance to etoposide (57\% vs $44 \%, P=.0009)$ and doxorubicin (73\% vs. $58 \%, P=.0324)$ and less intermediate or extreme resistance to cisplatin $(44 \%$ vs $54 \%, P=.0125)$, to carboplatin ( $47 \%$ vs $57 \%, P=.0129)$, to taxol (47\% vs $57 \%$, $P=.0056)$, and to gemcitabine (78\% vs $87 \%, P=.0108)$. Fewer epithelial growth factor receptor-positive tumors were extremely drug resistant to cisplatin $(13 \%$ vs $26 \%, P=.0074)$ and carboplatin $(13 \%$ v. 30\%, $P=.0008)$.

Conclusions: Multi-drug chemotherapy resistance in non-small cell lung cancer tumor cultures is common, and associations between molecular markers and in vitro chemotherapy resistance are noted. Clinical validation through integration of such testing into clinical trials seems warranted.

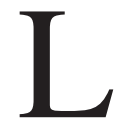

ung cancer is the most common cause of cancer-related mortality. It will account for an estimated 162,460 deaths in 2006 , and approximately 174,470 new cases were expected this year. Although surgical therapy remains the primary treatment for resectable disease, the composite 5-year survival is only 


\author{
Abbreviations and Acronyms \\ ANITA = Adjuvant Navelbine International Trialist \\ Association trial \\ ASCO = American Association of Clinical Oncology \\ CALGB $=$ The Cancer and Leukemia Group B \\ ECOG = Eastern Cooperative Oncology Group \\ EDR = extreme drug resistant \\ EGFR = epithelial growth factor receptor \\ IALT = International Adjuvant Lung Cancer Trial \\ IDR = intermediate drug resistant \\ LDR = low drug resistant \\ MDR1 = multiple drug resistance \\ NSCLC $=$ non-small cell lung cancer \\ PCI = percent colony inhibition
}

50\%. ${ }^{1}$ Platinum-based adjuvant chemotherapy, which has been reported to improve survival $(4 \%-15 \%)$ for some patients with early-stage non-small cell lung cancer (NSCLC), is now recommended for those with good performance status and completely resected stage IB-IIIA disease, ${ }^{2-5}$ yet only one study ${ }^{3}$ showed benefit for patients with stage IB disease. Accordingly, the majority of patients endure a predictable toxicity from empiric chemotherapy without a survival advantage.

In contrast to targeted therapeutic agents, such as trastuzumab inhibition of epithelial growth factor receptor (EGFR), which may hold promise toward patient-tumor specific treatment, ${ }^{6}$ empiric platinum-based chemotherapy regimens represent a "hit or miss" approach in hopes for a therapeutic benefit despite the calculable risk of significant toxicity.

By avoiding a particular drug by testing a specific tumor's phenotypic chemotherapy resistance, unnecessary toxicity may be avoided. In vitro chemotherapy resistance testing of fresh human tumor cells may accurately (90\%) predict that an agent may be ineffective, whereas chemotherapy sensitivity testing is less accurate (60\%) largely because it is impossible to predict the impact of important host and tumor biologic determinants affecting a drug's clinical efficacy. ${ }^{7}$ For some solid tumors, ${ }^{8-10}$ clinical unresponsiveness to antineoplastic agents correlates with in vitro chemotherapy resistance, yet little correlative data exist for NSCLC.

Recently, the prevalence of chemotherapy resistance in resected NSCLC tumor cultures to several individual antineoplastic drugs was reported. ${ }^{11}$ In an effort to further characterize in vitro chemotherapy resistance patterns in NSCLC, agents used clinically as platinum-based doublets were analyzed for simultaneous in vitro 2-drug resistance, and the association of chemotherapy resistance in NSCLC was compared with DNA ploidy and examined for correla- tions with molecular markers that may act as surrogates to predict tumor chemotherapy resistance.

\section{Materials and Methods NSCLC Specimens}

Between September 1989 and November 2005, 4571 fresh NSCLC tumor surgical biopsy specimens from 409 institutions were analyzed for chemotherapy resistance testing by Oncotech, Inc, Tustin, California. Since 1989, evaluability of submitted specimens averaged $81 \%$ (range $60 \%-90 \%$ ). The submitting institution determined tumor histologic type. Information regarding tumor histologic subtype, stage, and prior chemotherapy were not routinely reported for all specimens. From the tumor database, queries were performed to determine the prevalence of chemotherapy resistance to antineoplastic agents commonly used in the treatment of NSCLC and to determine whether any correlations existed between chemotherapy resistance patterns and DNA ploidy. For patient NSCLC tumors with known adenocarcinoma histology, the molecular markers p53 and EGFR were evaluated. All patient identifiers are dissociated from this database, and per institutional review board approval, patient consent was waived.

\section{The Extreme Drug Resistant (EDR) Assay}

Fresh tumor specimens are mechanically and enzymatically disaggregated into single cells and small cellular aggregates and cultured in a cellular proliferation assay described previously in detail. $^{7}$ Tumor cultures were exposed to single chemotherapeutic agents: cisplatin, carboplatin, etoposide, doxorubicin, topotecan, paclitaxel, docetaxel, vinorelbine, or gemcitabine in duplicate or triplicate assays at final concentrations 5 to 10 times higher than expected in vivo peak plasma levels. After a 72-hour exposure, 5 $\mu \mathrm{Ci}$ of ${ }^{3} \mathrm{H}$-thymidine (Amersham Biosciences, Piscataway, NJ) is added and incubation is continued for an additional 48 hours. Cell suspensions are harvested and cellular proliferation is determined by ${ }^{3} \mathrm{H}$-thymidine incorporation into DNA and measured by scintillation counting (Beckman-Coulter, Inc, Fullerton, Calif). Positive controls (supralethal cisplatin resulting in $100 \%$ cell death) prepared in duplicate and negative controls (media exposed only) prepared in quadruplicate are used to determine the percent colony inhibition (PCI) by an individual drug compared with mediaexposed cultures correcting for positive controls.

From a historical database of over 140,000 human tumors of varied histologic type submitted for the EDR assay, the PCI values are compared with the median PCI of the entire population for any given drug tested. Tumors exhibiting PCI values $1 \mathrm{SD}$ above the population median are defined as low drug resistant (LDR); tumors with PCI values between the population median and 1 SD below the median are defined as intermediate drug resistant (IDR); and tumors with PCI values that are 1 SD below the median PCI are defined as EDR.

\section{DNA Ploidy and S-phase Analyses by Flow Cytometry} Analyses were performed on nuclear suspensions prepared from fresh tissue as described ${ }^{12}$ using a Becton-Dickenson FACScan flow cytometer (Becton-Dickinson, Franklin Lakes, NJ) with DNA cell cycle and S-phase parameters programmed with ModFit LT software (Variety Software House, Inc, Topsham, Maine). 


\section{Immunohistochemistry}

From paraffin-embedded tissue sections, both mutant and wildtype isoforms of p53 were detected with the DO-1 monoclonal antibody (Santa Cruz Biotechnology, Inc, Santa Cruz, Calif) followed by automated immunostaining on a Ventana Benchmark (Vantana Medical Systems, Inc, Tucson, Ariz) or the BioGenex i6000 (BioGenix, Inc, San Ramon, Calif) instrument. The 3,3'diaminobenzidine/horse radish peroxidase reagent (Genetex, Inc, San Antonio, Tex) was used for secondary detection. Positive staining was defined as more than $20 \%$ of cells exhibiting nuclear staining. ${ }^{13}$

Automated measurement of EGFR expression was detected with the 31G7 monoclonal antibody (Invitrogen Corp, Carlsbad, Calif) after antigen retrieval with $0.25 \%$ trypsin. Positive staining was defined by calculating the histoscore, which equals the product of the percent EGFR positive cells times the staining intensity scored on a scale from zero (no staining) to 3 (intense staining) as described. ${ }^{14}$ An EGFR histoscore of 120 or more is considered positive.

\section{Statistical Analysis}

Patient tumor cultures were tested against a panel between 3 and 9 individual drugs. From cultures tested with agents used in the 4 clinically relevant platinum-based doublets-carboplatin plus taxol; cisplatin plus navelbine; cisplatin plus docetaxel; or cisplatin plus gemcitabine-concordant resistance patterns were determined by plotting PCI values for individual tumors tested with the first drug (ordinate) versus the same tumor tested against the second drug (abscissa). Assuming that some frequency of EDR, IDR, and LDR to any given drug exist in a population of separate tumors tested against 1 drug, if a set of tumors is tested against 2 independent drugs, then a $3 \times 3$ matrix can be created in which 9 possible resistance patterns are possible for the population. Within this population, each tumor has a unique resistance to each agent; for example, one subset of tumors will be EDR to both drugs and a subset will be LDR to both drugs. Seven other combinations of EDR, IDR, and LDR are possible. From this matrix, subpopulations exhibiting any EDR plus IDR combination to both drugs tested and those exhibiting EDR or IDR to at least one agent were calculated. Correlation coefficients from scatter plot analysis using linear regression analysis were performed with the Spotfire statistical package (Spotfire, Inc, Sommerville, Mass) to illustrate tumor resistance patterns for any given doublet pair analyzed.

Potential associations between drug resistance to a single agent and DNA ploidy or the molecular markers p53 and EGFR were also abstracted from the database with Spotfire and analyzed with SPSS software (SPSS, Inc, Chicago, Ill) by $\chi^{2}$ testing.

\section{Results}

Single-agent Chemotherapy Resistance Profiles

Drug-resistant profiles are shown in (Figure 1) for NSCLC tumor cell cultures exposed to frequently used chemotherapy agents. The frequency of EDR ranged from 13\% (202/ 1531) for topotecan and $13 \%(280 / 1351)$ for navelbine to $61 \%(917 / 1513)$ for gemcitabine. EDR tumors were noted in 485 (16\%) of 3042 cultures exposed to cisplatin, in 518 (23\%) of 2283 cultures exposed to carboplatin, in 432

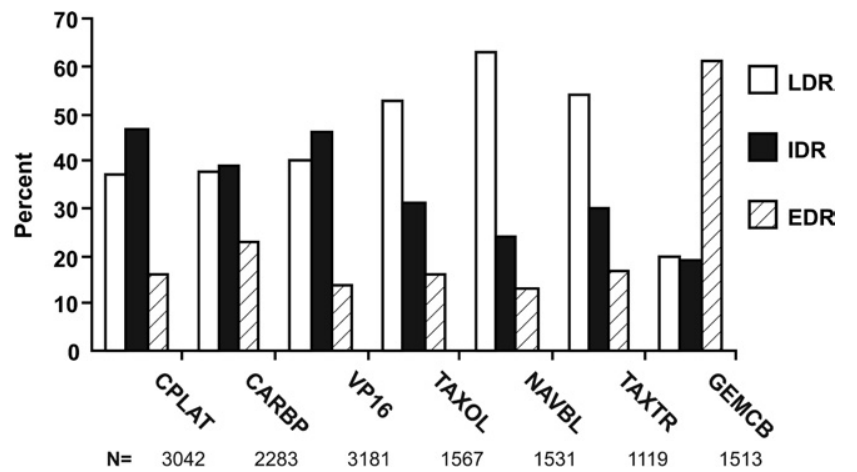

Figure 1. The frequency of chemotherapy resistance for NSCLC tumor cultures exposed to cisplatin (CPLAT), carboplatin (CARBP), etoposide (VP16), paclitaxel (TAXOL), vinorelbine (NAVBL), docetaxel (TAXTR), and gemcitabine (GEMCB) are shown as percentages. Low drug resistance (LDR) is inhibition of growth $1 \mathrm{SD}$ above the population median; intermediate drug resistance (IDR) is inhibition between the population median and 1 SD below the median; and extreme drug resistant (EDR) tumors are 1 SD below the median population.

(14\%) of 3181 cultures exposed to etoposide, in $392(16 \%)$ of 2485 cultures exposed to taxol, in $186(17 \%)$ of 1119 cultures exposed to docetaxel, and in 337 (22\%) of 1567 cultures exposed to doxorubicin.

\section{Paired Analysis}

NSCLC tumor cultures assayed separately against platin agents (cisplatin plus carboplatin) and vinca alkaloids (vincristine plus vinorelbine [Navelbine]) were analyzed for concordant resistant patterns. For cisplatin and carboplatin 1099 specimens were tested separately to both agents and showed similar distributions of drug resistance $(R=0.76)$. For the vinca alkaloids, 28 cultures were assayed with both agents and resistance profiles correlated $(R=0.92)$ (Figure 2$)$. Little correlation was noted on scatter plots for the paired combinations carboplatin versus paclitaxel and the drug combinations tested with cisplatin: navelbine, docetaxel, or gemcitabine. For 1523 tumors separately tested with carboplatin versus paclitaxel $(R=0.189), 80(5 \%)$ were EDR and 393 (26\%) were LDR to both drugs. Combined IDR and EDR tumors were observed in $422(28 \%)$ of the population, and $1130(74 \%)$ were IDR or EDR to at least one agent (Figure 3, A). In 1314 cultures tested for cisplatin versus navelbine $(R=0.018), 32(2 \%)$ were EDR and 418 (32\%) were LDR to both. Combined IDR and EDR tumor cultures comprised 263 (20\%) of the population, and 896 $(68 \%)$ were IDR or EDR to at least one drug (Figure 3, $B)$. For cisplatin versus docetaxel, of 741 cultures tested $(R=0.182), 35(5 \%)$ were EDR and (32\%) were LDR to both. Combined IDR and EDR tumor cultures comprised 


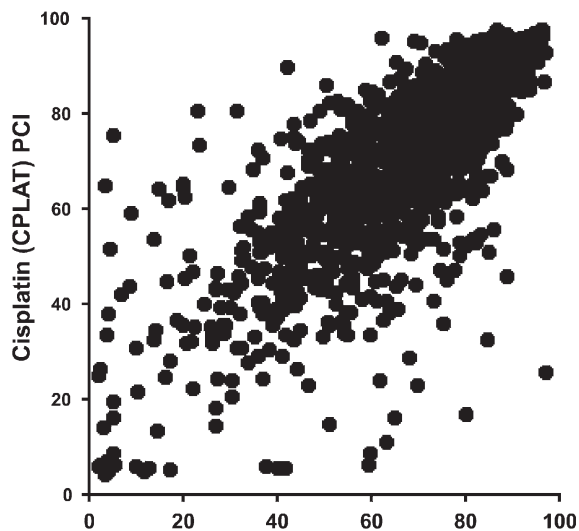

A Carboplatin (CARBO) PCI

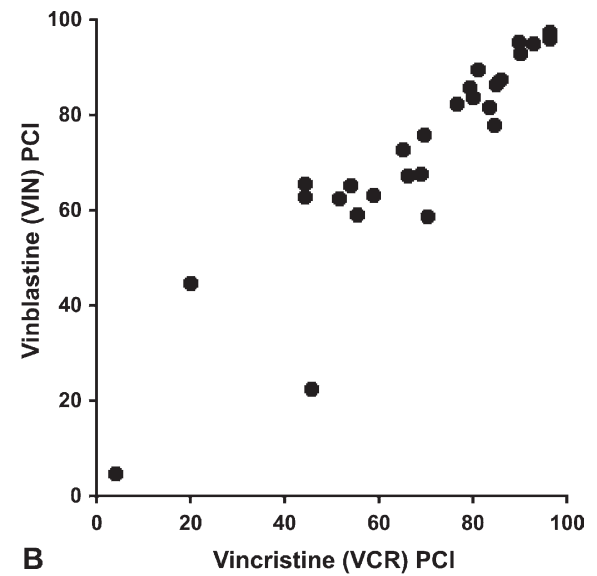

Figure 2. Correlation and concurrent chemotherapy resistance illustrating congruent patterns for NSCLC tumor cultures exposed in separate assays to like agents, cisplatin (CPLAT) and carboplatin (CARBO) ( $\mathrm{N}=1099, R=0.76)$ (A) or to vinblastine (VIN) and vincristine (VCR) ( $\mathrm{N}=28, R=0.92)(\mathrm{B})$, are shown as percent colony inhibition (PCI) for each drug tested. Each data point (jittered for clarity) represents the combined in vitro response for a single tumor culture.
$202(27 \%)$ of the population, and $507(68 \%)$ were resistant to at least one (Figure 3,C). For cisplatin versus gemcitabine, of 994 cultures tested $(R=0.09), 136$ (14\%) were EDR and $110(11 \%)$ were LDR to both. Combined IDR and EDR tumor cultures comprised 439 (44\%) of the population and $884(89 \%)$ were resistant to at least one (Figure 3,D).
Chemotherapy Resistance and Ploidy

An association between chemotherapy resistance and diploid versus nondiploid tumors was examined. LDR compared with combined IDR and EDR tumors showed that aneuploid tumors were more likely to be resistant to etoposide, $218(53 \%)$ of 415 versus 59 (36\%) of $167(P$ $=.0002)$, and topotecan, $177(48 \%)$ of 369 versus 56
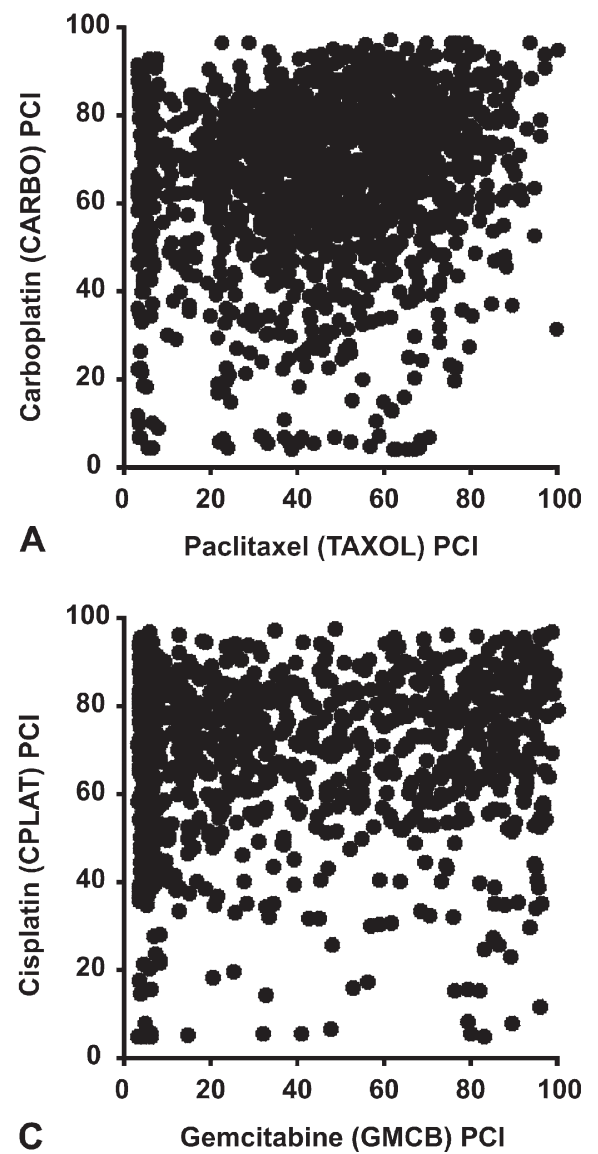
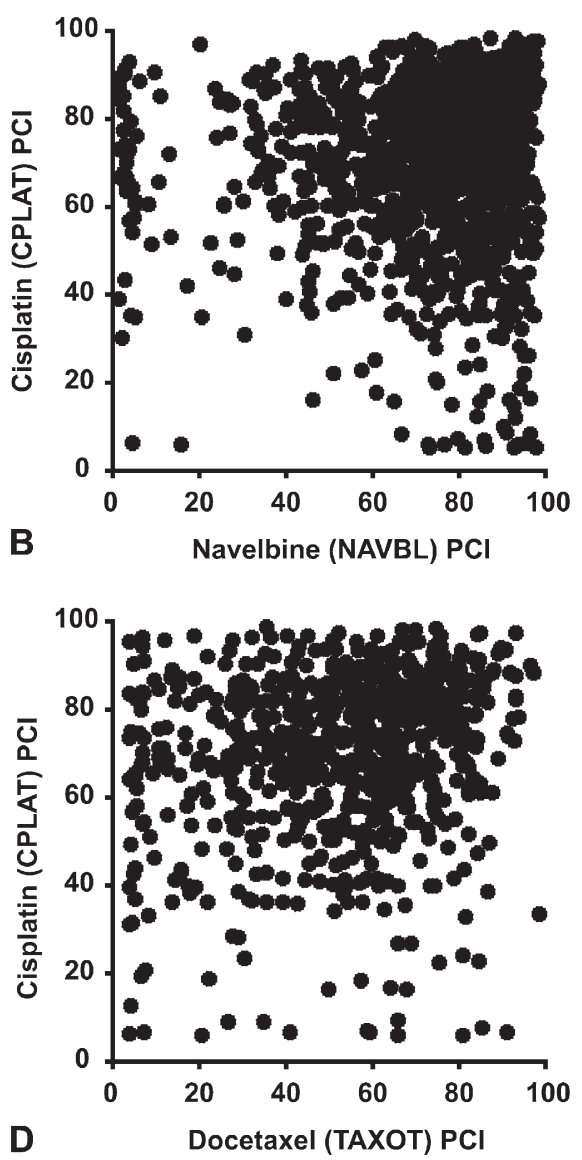

Figure 3. Concurrent chemotherapy resistance patterns for NSCLC tumor cultures exposed in separate assays to one of four standard platinum-based chemotherapy doublets. A, Carboplatin (CARBO) and paclitaxel (TAXOL) (N = 1523, $R=0.189$ ); $B$, cisplatin (CPLAT) and navelbine (NAVBL) (N = 1314, $R=$ 0.018); $C$, cisplatin (CPLAT) and gemcitabine (GMCB) ( $N=741, R=0.182)$; and D, cisplatin (CPLAT) and docetaxel (TAXOT) ( $N=994, R=0.09$ ) are shown for each pair as percent colony inhibition (PCI) for each drug tested. Each data point (jittered for clarity) represents the combined in vitro response for a single tumor culture. 
TABLE 1. Association between DNA ploidy and in vitro chemotherapy resistance

\begin{tabular}{|c|c|c|c|c|c|c|c|c|c|}
\hline \multirow[b]{4}{*}{ Agent } & \multicolumn{8}{|c|}{ DNA ploidy } & \multirow{4}{*}{$\frac{P \text { value }}{\chi^{2}}$} \\
\hline & \multicolumn{4}{|c|}{ Diploid } & \multicolumn{4}{|c|}{ Aneuploid } & \\
\hline & \multicolumn{2}{|c|}{ LDRT } & \multicolumn{2}{|c|}{ IDR‡ + EDR§ } & \multicolumn{2}{|c|}{ LDR } & \multicolumn{2}{|c|}{ IDR + EDR } & \\
\hline & $\mathbf{N}$ & $\%$ & $\mathbf{N}$ & $\%$ & $\mathbf{N}$ & $\%$ & $\mathbf{N}$ & $\%$ & \\
\hline Cisplatin & 76 & 46 & 91 & 54 & 209 & 54 & 181 & 46 & .0804 \\
\hline Carboplatin & 102 & 53 & 92 & 47 & 214 & 49 & 221 & 51 & .4334 \\
\hline Etoposide & 108 & 65 & 59 & 35 & 197 & 47 & 218 & 53 & .0002 \\
\hline Doxorubicin & 11 & 46 & 56 & 36 & 24 & 42 & 33 & 58 & .7571 \\
\hline Topotecan & 101 & 64 & 56 & 36 & 192 & 52 & 177 & 48 & .0094 \\
\hline Taxol & 98 & 49 & 101 & 51 & 202 & 45 & 248 & 55 & .3046 \\
\hline Taxotere & 73 & 54 & 63 & 46 & 190 & 57 & 144 & 43 & .5250 \\
\hline Navelbine & 132 & 73 & 50 & 27 & 282 & 66 & 145 & 34 & .1164 \\
\hline Gemcitabine & 20 & 12 & 149 & 88 & 75 & 19 & 317 & 81 & .0345 \\
\hline
\end{tabular}

$* P$ value for $\chi^{2}<.05$ is significant. †Low drug resistance. $\ddagger$ Intermediate drug resistance. $\S$ Extreme drug resistance.

(36\%) of $157(P=.0094)$, whereas aneuploid tumors were less resistant to gemcitabine, $149(88 \%)$ of 169 versus $317(81 \%)$ of $392(P=.0345)$. Aneuploid tumors were relatively less resistant to cisplatin and taxotere and more resistant to carboplatin, doxorubicin, taxol, and navelbine, but differences were not statistically significant (Table 1).

\section{Chemotherapy Resistance and Markers p53 and EGFR}

Positive versus negative p53 staining was compared with LDR versus IDR plus EDR tumors. In p53-positive tumors compared with p53-negative tumors, cultures were more resistant to etoposide, 169 (57\%) of 295 versus 171 (44\%) of $385(P=.0009)$ and to doxorubicin, $66(73 \%)$ of 90 versus $53(58 \%)$ of $91(P=.0324)$. Less chemotherapy resistance was noted in p53-positive tumors for cisplatin in 127 (44\%) of 288 versus $200(54 \%)$ of $371(P=.0125)$, for carboplatin in $136(47 \%)$ of 288 versus $213(57 \%)$ of 374
$(P=.0129)$, for taxol in $148(47 \%)$ of 315 versus 234 $(57 \%)$ of $408(P=.0056)$, and for gemcitabine in 185 (78\%) of 236 versus $283(87 \%)$ of $327(P=.0108)$. No statistical difference was noted for topotecan, taxotere, or navelbine (Table 2).

LDR plus IDR versus EDR chemotherapy resistance was compared to low versus high EGFR histoscores, defined as the product of staining intensity $\times$ the number of positively stained cells. High EGFR histoscores were associated with decreased EDR for cisplatin in $23(13 \%)$ of 183 versus 19 (26\%) of $72(P=.0074)$, for carboplatin in $26(13 \%)$ of 203 versus $24(30 \%)$ of $81(P=.0008)$, and trend toward significance for topotecan in $32(18 \%)$ of 182 versus $20(27 \%)$ of 75 $(P=.0994)$ was noted. Increased EDR for gemcitabine was observed in $130(67 \%)$ of 194 versus $40(52 \%)$ of $77(P=$ .0207) in tumors with high EGFR histoscores. No statistically significant differences were observed for etoposide, doxorubicin, taxol, taxotere, or navelbine (Table 3).

TABLE 2. Association between p53 and in vitro chemotherapy resistance for NSCLC of adenocarcinoma histology

\begin{tabular}{|c|c|c|c|c|c|c|c|c|c|}
\hline \multirow{3}{*}{ Agent } & \multicolumn{8}{|c|}{ p53 by IHC } & \multirow{3}{*}{$\frac{P \text { value }}{\chi^{2}}$} \\
\hline & \multicolumn{4}{|c|}{ p53 Negative } & \multicolumn{4}{|c|}{ p53 Positive } & \\
\hline & \multicolumn{2}{|c|}{ LDRT } & \multicolumn{2}{|c|}{ IDR‡ + EDR§ } & \multicolumn{2}{|c|}{ LDR } & \multicolumn{2}{|c|}{ IDR + EDR } & \\
\hline Cisplatin & 171 & 46 & 200 & 54 & 161 & 56 & 127 & 44 & .0125 \\
\hline Carboplatin & 161 & 43 & 213 & 57 & 152 & 53 & 136 & 47 & .0129 \\
\hline Topotecan & 172 & 55 & 141 & 45 & 109 & 52 & 99 & 48 & .5677 \\
\hline Taxol & 174 & 43 & 234 & 57 & 167 & 53 & 148 & 47 & .0056 \\
\hline Taxotere & 144 & 55 & 119 & 45 & 114 & 57 & 85 & 43 & .5871 \\
\hline Navelbine & 238 & 67 & 117 & 33 & 186 & 69 & 82 & 31 & .5315 \\
\hline Gemcitabine & 44 & 13 & 283 & 87 & 51 & 22 & 185 & 78 & .0108 \\
\hline
\end{tabular}

$* P$ value for $\chi^{2}<.05$ is significant. †Low drug resistance. $\ddagger$ Intermediate drug resistance. $\S$ Extreme drug resistance. 
TABLE 3. Association between EGFR and in vitro chemotherapy resistance for NSCLC of adenocarcinoma histology

\begin{tabular}{|c|c|c|c|c|c|c|c|c|c|}
\hline \multirow[b]{4}{*}{ Agent } & \multicolumn{8}{|c|}{ EGFR by IHC } & \multirow{4}{*}{$\frac{P \text { value }}{\chi^{2}}$} \\
\hline & \multicolumn{4}{|c|}{ EGFR histoscore low $<120$} & \multicolumn{4}{|c|}{ EGFR histoscore high $\geq 120$} & \\
\hline & \multicolumn{2}{|c|}{ LDR† + IDR } & \multicolumn{2}{|c|}{ EDR§ } & \multicolumn{2}{|c|}{ LDR + IDR } & \multicolumn{2}{|c|}{ EDR } & \\
\hline & $\mathbf{N}$ & $\%$ & $\mathbf{N}$ & $\%$ & $\mathbf{N}$ & $\%$ & $\mathbf{N}$ & $\%$ & \\
\hline Cisplatin & 53 & 74 & 19 & 26 & 160 & 87 & 23 & 13 & .0074 \\
\hline Carboplatin & 57 & 70 & 24 & 30 & 177 & 87 & 26 & 13 & .0008 \\
\hline Etoposide & 67 & 92 & 6 & 8 & 160 & 87 & 24 & 13 & .2774 \\
\hline Doxorubicin & 5 & 71 & 2 & 29 & 11 & 79 & 3 & 21 & .7171 \\
\hline Topotecan & 55 & 73 & 20 & 27 & 150 & 82 & 32 & 18 & .0994 \\
\hline Taxol & 66 & 79 & 18 & 21 & 167 & 82 & 37 & 18 & .5183 \\
\hline Taxotere & 63 & 94 & 4 & 6 & 163 & 92 & 15 & 8 & .5216 \\
\hline Navelbine & 79 & 95 & 4 & 5 & 178 & 91 & 17 & 9 & .2603 \\
\hline Gemcitabine & 37 & 48 & 40 & 52 & 64 & 33 & 130 & 67 & .0207 \\
\hline
\end{tabular}

$* P$ value for $\chi^{2}<.05$ is significant. $\dagger$ Low drug resistance. $\ddagger$ Intermediate drug resistance. $\S$ Extreme drug resistance.

\section{Discussion}

\section{In Vitro Chemotherapy Resistance Versus Chemosensitivity Testing}

The clinical utility of in vitro chemotherapy sensitivity and resistance assays to estimate clinical response to chemotherapy has been debated for nearly a decade, and in recent years some oncologists, having continued skepticism, may not fully appreciate some important historical developments that led to the distinction between chemosensitivity and chemoresistance. The latest review and technology assessment published by the American Association of Clinical Oncology (ASCO) is an example of recent publications that were considered biased and nearsighted by some, failing to acknowledge several shortcomings of older methods and defining salient differences between sensitivity and resistance testing. ${ }^{15-18}$ Advances in cell culture technology, test availability, reliability, and reproducibility attest to the robust nature of some chemotherapy-resistant assays and prompted the Medicare approval of chemotherapy resistance (not sensitivity) testing. ${ }^{19}$ Two types of assays are described below to illustrate differences between chemotherapy resistance and sensitivity.

Older clonogenic assays relied on brief exposures to chemotherapy at or below peak serum concentrations, frequently used cell culture techniques permitting growth of stromal elements, required long incubation times, which limited clinical usefulness, and often used inaccurate manual cell-counting methods. Assumptions that in vitro tumor sensitivity equates with clinical response accounts for neither tumor factors of anatomic permeability and vascularity nor host factors of absorption, metabolism, activation, or elimination.

In contrast, the EDR assay is a cellular proliferation assay in which tumors are exposed for 120 hours to suprapharmacologic doses of chemotherapy up to 10 -fold higher than peak plasma concentrations. Stromal cell growth is inhibited by cell platting on soft agarose, and cellular proliferation is reliably measured by ${ }^{3} \mathrm{H}$-thymidine incorporation into DNA. When the relative cellular growth of a specific culture is compared with well over 140,000 human tumors exposed to the same drug, tumor growth inhibition 1 SD below the median inhibition identifies resistant tumors without reference to clinical sensitivity. Comparing clinical response to chemotherapy with an independent tumor set of 450 human tumor cell cultures, 20 of which were NSCLC, the ability of the EDR assay to detect tumor resistance to a particular chemotherapeutic agent was over $99 \%$ specific. $^{7}$ Recently, we have rekindled an interest in the clinical application of chemotherapy resistance assays not only to help predict an observed response to adjuvant chemotherapy, but in hopes of avoiding unnecessary toxicities related to potentially ineffective cytotoxic agents.

\section{Adjuvant Chemotherapy in Resected NSCLC}

Enthusiasm to administer platinum-based adjuvant chemotherapy after complete resection of stage IB-IIIA NSCLC has been fueled from the results of 4 randomized clinical trials. Controversy regarding the universal application of adjuvant chemotherapy after resection of stage IB disease continues. In the International Adjuvant Lung Cancer Trial (IALT), patients with stage IA-IIIA disease received cisplatin plus etoposide $(57 \%)$ or a vinca alkaloid $(43 \%)$. Although an overall $4 \%$ increase in 5-year survival was noted, only patients with stage IIIA disease benefited. Grade 4 toxicity occurred in $24 \%$, and non-dose dependent lethal platinum toxicity occurred in up to $2.4 \%$ of patients. ${ }^{2}$

Both the National Cancer Institute of Canada Clinical Trial Group-JBR.10 trial and the Adjuvant Navelbine International Trialist Association (ANITA) trial used the cisplatin plus vinorelbine doublet for adjuvant therapy after complete resection. JBR.10 included patients with stages 
IB-II disease, but a survival benefit occurred only in patients with stage II disease. Grade 3 or 4 neutropenia occurred in $73 \%$ of patients in the chemotherapy arm. In the ANITA trial, survival advantage with adjuvant chemotherapy was noted for patients with stage II-IIIA disease, but no observed survival benefit was noted in patients with stage IB disease. ${ }^{4,5}$

The Cancer and Leukemia Group B (CALGB)- 9633 trial randomized only patients with stage IB (T2 N0) disease either to adjuvant carboplatin plus paclitaxel or to observation alone after complete resection. These data, presented in abstract form at ASCO in 2005, are yet to be published otherwise. They reported an improvement in overall survival among the adjuvant chemotherapy patient arm of the study of $12 \%$ at 4 years' follow-up. Grade 4 neutropenia occurred in $36 \%$, and $45 \%$ of patients either did not complete therapy or required dose reductions. This recent trial is alone in reporting a demonstrable survival benefit for adjuvant chemotherapy in patients with stage IB disease. ${ }^{3}$ Subsequent reanalysis of the data from this trial suggests that the study design was underpowered to definitely suggest a long-term survival benefit of adjuvant chemotherapy for stage IB disease. ${ }^{20}$ These more recent findings provoke continued skepticism with the utility of presently available empiric adjuvant chemotherapy for completely resected stage IB disease.

\section{Multiple Drug Chemotherapy Resistance}

In this study, the resistance of NSCLC tumor cultures exposed to several individual chemotherapy agents of clinical relevance was analyzed. The results are consistent with our previously reported prevalence of chemotherapy resistance in both NCSLC tumor cultures ${ }^{11}$ and other solid tumors. ${ }^{14,21}$ Despite potential limitations of not exposing tumors to two drugs simultaneously to test for chemotherapeutic agent synergy, these data illustrate that tumors are frequently resistant to at least one agent in chemotherapy doublet regimens commonly used throughout North America and Europe today. Even though the frequency of tumors exhibiting extreme chemotherapy resistance to both agents is less impressive, the frequency of extreme or intermediate resistance to at least one chosen agent is alarmingly high, suggesting that patients may benefit from only one drug with empiric "doublet chemotherapy" selection. Accordingly, Mehta and associates ${ }^{8}$ examined this phenomenon in breast cancer and found that the median time to disease progression and survival was significantly shorter for patients treated with any combination of agents exhibiting either extreme or intermediate in vitro drug resistance in comparison with patients having tumors with low in vitro resistance to both drugs. In vitro drug resistance correlated with a shorter survival similar to that associated with advanced stage or positive lymph node status. It is interesting to note that in our study, tumor resistance observed in doublet analysis is in parallel with the observed clinical response reported in the JBR.10 and ANITA trials, where the greatest survival advantage is reported with the cisplatin plus vinorelbine doublet. ${ }^{4,5}$

\section{DNA Ploidy, Molecular Markers, and Chemotherapy Resistance}

We report an association between tumor chemotherapy resistance and DNA ploidy for some antineoplastic agents. Aneuploid tumors were likely to be less resistant to gemcitabine, yet were more resistant to etoposide and topotecan. The potential clinical relevance of this association for these drugs is interesting but obscure. Other investigators have also identified a possible relationship between tumor ploidy and chemotherapy resistance. Doubre and associates ${ }^{22}$ reported a 3-fold increase in levels of multiple drug resistance protein (MDR1) in 84 previously untreated aneuploid NSCLC tumors and found that DNA aneuploidy resulted from an increased gain of chromosome 16 where the MDR1 gene is located. Volm and colleagues ${ }^{23}$ analyzed 240 patients with NSCLC having aneuploid tumors with more than one stem line. In vitro chemotherapy resistance was associated with an observed decreased survival in patients undergoing chemotherapy having aneuploid tumors compared with patients with diploid and in vitro sensitive tumors.

Our investigation has also identified a possible association between p53 expression and chemotherapy drug resistance. We found that $\mathrm{p} 53$-positive tumors were more often less resistant to cisplatin, carboplatin, paclitaxel, and gemcitabine, but were more often associated with increased resistance to etoposide and doxorubicin. This association for cisplatin in vitro resistance in p53-positive tumors is inconsistent with clinical results from others ${ }^{24,25}$ in which $26 \%$ patients with p53-positive tumors in chemotherapy naive advanced NSCLC responded to cisplatin versus $57 \%$ of patients with $\mathrm{p} 53$-negative tumors. This discrepancy may be related to the ability of suprapharmacologic doses of some chemotherapeutic agents to overcome the suppressor gene checkpoint imparted by p53. Interestingly, d'Amico and coworkers ${ }^{26}$ showed that female patients with stage I lung cancer and p53-positive tumors had a decreased survival of $49 \%$ compared with $77 \%$ of women with p53negative tumors.

Increasing interest in the prognostic importance of EGFR expression related to targeted therapy for NSCLC stimulated us to examine this molecular marker with regard to resistance to "standard" chemotherapy agents for this disease. Onn and associates ${ }^{27}$ reported a trend toward decreased survival from 84.4 months to 44.2 months among 111 patients with stage 1 NSCLC who had synchronous expression of EGFR and the HER2-neu protein. The finding of EGFR gene polymorphisms measured by polymerase chain reaction-restriction fragment polymorphism has been 
evaluated in advanced colorectal cancer and has been found to be associated with an increased likelihood of disease progression with 5-fluorouracil/oxaliplatin chemotherapy when the increased gene copy number was identified. ${ }^{28}$ Observations from our study revealed that increased EGFR expression, measured by immunohistochemistry, is associated with a decreased in vitro resistance to platinum agents and increased in vitro resistance to gemcitabine. Our study lacks clinical correlation and distinction between EGFR expression levels, the presence of specific mutations determined by polymerase chain reaction, or measurements of gene copy number by florescent in situ hybridization. Comparisons of these assay modalities may warrant additional study. Further analysis of associations between EGFR and chemotherapy resistance should be considered for earlystage patients based on the decreased survival noted for EGFR-positive tumors in patients with stage I disease. ${ }^{27}$

\section{The Cellular Proliferation Chemotherapy Resistance Assay and the Relationship to the Cancer Stem Cell Hypothesis}

In regeneration of normal (nontransformed) tissue, quiescent stem cells are activated to enter the cell cycle and the formation of new replacement tissue occurs in response to environmental stress. The cancer stem cell hypothesis states that the cancer-initiating cell is a transformed tissue stem cell, which retains the essential property of self-renewal through the activity of MDR transporters. ${ }^{29}$ In normal tissue, the genetic regeneration program is turned off after tissue restoration once homeostasis is restored. Unlike normal tissues, homeostasis in progenitor cancer cells is abnormal. Thus, tumor cells can overcome anticancer therapy not only by the activation of mechanisms specific for transformed cells, but also by taking advantage of a normal tissue regeneration genetic program. ${ }^{30}$

Human tumors are composed of nonhomogeneous cell populations including cancer stem cells and transformed progenitor cells. These heterogeneous populations are all represented in cell culture conditions used in the EDR assay and include subpopulations of cancer stem cells that constitutively express the MDR phenotype and are inherently chemotherapy resistant. An example of how the EDR assay can predict clinical chemotherapy resistance and may be related to the proliferation of cancer stem cells is noted in a study of breast cancer tumor cultures that exhibited an in vitro chemotherapy resistance to both paclitaxel and doxorubicin. ${ }^{14}$ Overall, MDR1 P-glycoprotein expression was shown to occur in $17 \%$ of these patients' tumors. When further stratified, tumors from chemotherapy naive patients showed an 11\% incidence of MDR1 expression compared with a 30\% incidence of MDR1 expression in tumors from previously treated patients. The clinical response to doxorubicin and paclitaxel was lower in the previously treated MDR1-positive groups than in the untreated MDR1negative groups. ${ }^{14}$

It should be noted that tumors might possess in vitro drug sensitivity because of an agents' anticancer activity or might exhibit resistance because of the tumors' phenotypic resistance to the agent. In the EDR assay, some agents may overcome unfavorable cellular transport kinetics or permeability barriers because of high drug concentration gradients that favor intracellular drug accumulation. These cells may exhibit in vitro sensitivity even if they are inherently resistant stem cells. This relates clinically to anticancer therapy and the cancer stem cell hypothesis where an agent might eliminate the main population (non-stem) cells, resulting in selection of cancer stem cells. It follows that transformation of this small population of cancer stem cells might ultimately result in clinical tumor progression, recurrence, or metastatic disease. A priori, a potential limitation of the ability of cellular proliferation assays to accurately predict clinical sensitivity, other than patient host factors, may be due to a relatively decreased potential for a small population of cancer stem cells to proliferate significantly relative to other tumor cells under tissue culture conditions of the EDR assay. Further studies on the relationship between in vitro chemotherapy resistance testing as it relates to the cancer stem cell hypothesis are ongoing.

\section{Randomized Trials and the Clinical Utility of Chemoresistance Testing}

$\mathrm{We}^{11}$ previously reported that extreme or intermediate in vitro chemotherapy resistance in NSCLC patient tumor cultures was prevalent, particularly for platinum agents $(63 \%-68 \%)$ and for gemcitabine (72\%). From the analysis of a larger database representing 4571 NSCLC fresh tumor cultures in this study, the incidence of resistance to platinum agents $(62 \%-63 \%)$ and gemcitabine $(80 \%)$ was similar. More important, we frequently found that many tumors exhibited simultaneous resistance to both agents used in four of the most commonly prescribed platinum-based chemotherapy doublets. These results raise concern regarding the widespread use of empiric adjuvant chemotherapy, where few patients may benefit, and in particular, consternation exists with the recommendation of adjuvant chemotherapy to patients with completely resected stage IB disease in whom a survival advantage is questionable. ${ }^{20}$

In patients with stage IV NSCLC, a randomized clinical trial by the Eastern Cooperative Oncology Group (ECOG) compared overall survival in patients treated with cisplatin plus gemcitabine, cisplatin plus docetaxel, or cisplatin plus paclitaxel in reference to the efficacy of carboplatin plus paclitaxel. An overall 19\% response rate and median survival of 7.9 months was observed with 1-year and 2-year survivals of $33 \%$ and $11 \%$, respectively. Neither response rates nor survival differed significantly with any regimen

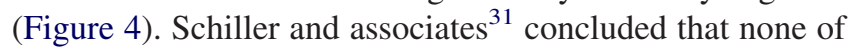



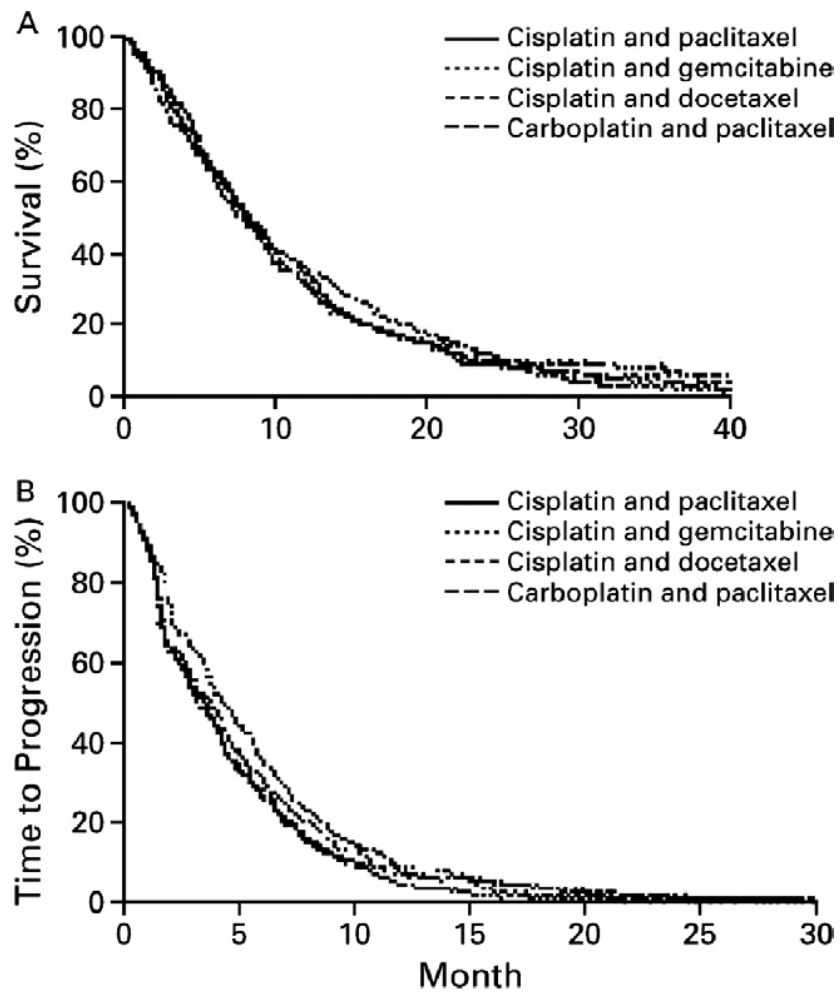

Figure 4. Kaplan-Meier estimates of overall survival $(A)$ and the time to progression of disease (B) in the ECOG study patients enrolled according to their assigned treatment (From Schiller JH, Harrington D, Belani CP, Langer C, Sandler A, Krook J, et al. Comparison of four chemotherapy regimens for advanced nonsmall-cell lung cancer. N Engl J Med. 2002;346:92-8. Reprinted by permission of the Massachusetts Medical Society. Copyright 2002 Massachusetts Medical Society. All rights reserved).

the four regimens offered a significant advantage over the others, yet the carboplatin plus paclitaxel doublet was chosen as the ECOG reference for future studies because of reduced toxicity. On the basis of these data showing similar clinical efficacy among several regimens, empiric selection of any doublet combination is without significant predictable value to the patient with regard to "doublet" efficacy. The application of tumor chemotherapy resistance testing therefore may be of clinical benefit.

Chemoresistance testing may help select a second drug for a platinum-based doublet by "deselecting" one agent on the basis of its in vitro chemotherapy resistance. Patients with metastatic disease and those undergoing "salvage" therapy may benefit most from chemotherapy deselection when one or more agents are considered, perhaps avoiding unnecessary toxicity from ineffective second- or third-line therapies.

Despite the intriguing possibilities of using chemoresistance testing to improve response and reduce toxicity re- lated to therapy, its use for the purpose of "assay directed" treatment is currently lacking clinical validation in NSCLC. The recent ASCO technology assessment ${ }^{15}$ has recommended that future studies be designed comparing empiric chemotherapy with resistance assay-directed chemotherapy. Despite these recommendations, it has been emphasized that despite the availability and reliability of chemotherapy resistance testing, the general medical oncology community has yet to integrate this concept into any cooperative group trial. ${ }^{18}$ In Weiands' rebuttal ${ }^{32}$ to this ASCO report, he suggests that the clinical utility of resistance testing be validated in a single-arm, noninterventional assay accuracy trial using two or more therapies known to have similar response rates to determine whether the assay is predictive of response. We agree with Weiand's assessment of this concept and would support such as trial for NSCLC.

Validation of the clinical utility of the EDR assay may be best achieved by correlating in vitro resistance to clinical outcome in patients considered for adjuvant chemotherapy who are eligible for enrollment in an existing adjuvant chemotherapy trial, or as a companion to a surgical trial with an adjuvant chemotherapy arm. The recently proposed ECOG 1505 phase III trial will randomize 1500 eligible patients with resected stage IB-IIIA NSCLC to adjuvant chemotherapy with the four most accepted platinum-based "doublets" with or with out bevacizumab. Drug resistance testing is well suited as a companion to this trial, irrespective of a potential bevacizumab response.

Recently, members at Duke University developed a genomic strategy to estimate prognosis in patients with resected stage I NSCLC using RNA micro array analysis. By developing a multiple gene expression profile, they created a lung metagene model and validated it with two independent tumor sets. The prognostic accuracy to predict recurrence and survival approached $90 \% .{ }^{33}$ These results have prompted the development of a CALGB trial designed to prospectively validate the lung metagene models' ability to differentiate low-risk from high-risk patients. Patients with completely resected stage IA NSCLC will be separated in a 1:2 ratio into a low-risk (observation only) group and high-risk group. High-risk patients will then be randomized to receive one of the four NSCLC adjuvant chemotherapy doublet regimens (similar to those proposed for ECOG 1505) or observation alone. Although this model may help distinguish between patients with a favorable prognosis who only require observation, half of the high-risk patients will be prescribed cytotoxic chemotherapy with an uncertain benefit. Despite these efforts to "target" therapy based on prognosis, empiric adjuvant chemotherapy is still applied in this study. Clinical observations of recurrence and survival in the high-risk patient arm, if correlated with chemotherapy resistance testing, may help further define the clinical utility of an in vitro chemoresistance testing assay. 
As the public continues to cry out to "wage the war on cancer," 34 identifying genomic profiles that predict prognosis, and using molecular marker assays to predict therapeutic response, developing clinically relevant studies for more selective therapies will become increasingly important. Integrating molecular testing and chemotherapy resistance assays into future adjuvant chemotherapy trials for NSCLC will facilitate avoiding yet another decade of pure empiricism in patient care. This paradigm shift appears necessary and justified.

\section{References}

1. American Cancer Society cancer facts and figures 2006. Atlanta: American Cancer Society; 2006. p. 13-5.

2. The International Adjuvant Lung Cancer Trial Collaborative Group. Cisplatin-based adjuvant chemotherapy in patients with completely resected non-small-cell lung cancer. N Engl J Med. 2004;350:351-60.

3. Strauss GM, Herndon J, Maddaus MA, et al. Randomized clinical trial of adjuvant chemotherapy with paclitaxel and carboplatin following resection in Stage IB non-small cell lung cancer (NSCLC): report of Cancer and Leukemia Group B (CALGB) Protocol 9633. ASCO Annual Meeting Proceedings, New Orleans. J Clin Oncol. 2004;22: 7019.

4. Winton TL, Livingston R, Johnson D, et al. A prospective randomised trial of adjuvant vinorelbine (VIN) and cisplatin (CIS) in completely resected stage 1B and II non small cell lung cancer (NSCLC) Intergroup JBR.10. N Engl J Med. 2005;352:2289-97.

5. Douillard J, Rosell R, Delena M, et al. ANITA: Phase III adjuvant vinorelbine $(\mathrm{N})$ and cisplatin $(\mathrm{P})$ versus observation (OBS) in completely resected (stage I-III) non-small-cell lung cancer (NSCLC) patients (pts): final results after 70-month median follow-up. On behalf of the Adjuvant Navelbine International Trialist Association. ASCO Annual Meeting Proceedings, Orlando [FL]. J Clin Oncol. 2005;23: 7013.

6. Langer CJ, Stephenson P, Thor A, et al. Trastuzumab in the treatment of advanced non-small cell lung cancer: is there are role? Focus on Eastern Cooperative Oncology Group Study 2598. J Clin Oncol. 2004;22:1180-7.

7. Kern D, Weisenthal L. Highly specific prediction of antineoplastic drug resistance with an in vitro assay using suprapharmacologic drug exposures. J Natl Cancer Inst. 1990;82:582-8.

8. Mehta R, Bomstein R, Yu I-R, et al. Breast cancer survival and in vitro tumor response in the extreme drug resistance assay. Breast Cancer Res Treat. 2001;66:225-37.

9. Loizzi V, Chan J, Osann K, et al. Survival outcomes in patients with recurrent ovarian cancer who were treated with chemoresistance assay-guided chemotherapy. Am J Obstet Gynecol. 2003;189:1301-7.

10. Holloway R, Mehta R, Finkler N, et al. Association between in vitro platinum resistance in the EDR assay and clinical outcomes for ovarian cancer patients. Gynecol Oncol. 2002;87:8-16.

11. d'Amato T, Landreneau R, McKenna R, et al. Prevalence of in vitro extreme chemotherapy resistance in resected non-small cell lung cancer. Ann Thorac Surg. 2006;81:440-6.

12. Hedley D, Clark G, Cornelisse C, et al. DNA cytometry consensus conference consensus review of the clinical utility of DNA cytometry in carcinoma of the breast. Breast Cancer Res Treat. 1993;28:55-9.

13. Stoetzer O, Munker R, Darsow M, Wilmanns W. P53-immunoreactive cells in benign and malignant effusions: diagnostic value using a panel of monoclonal antibodies and comparison with CEA-staining. Oncol Rep. 1999;6:455-8.

14. Mechetner E, Kyshtoobayeva A, Zonis S, et al. Levels of multidrug resistance (MDR1) P-glycoprotein expression by human breast cancer correlate with in vitro resistance to taxol and doxorubicin. Clin Cancer Res. 1998;4:389-98.

15. Schrag D, Garewal H, Burstein H, et al. American Society of Clinical Oncology technology assessment: chemotherapy sensitivity and resistance assays. J Clin Oncol. 2004;22:3631-8.
16. Samson D, Seidenfeld J, Ziegler K, et al. Chemotherapy sensitivity and resistance assays: a systematic review. J Clin Oncol. 2004;22:3618-30.

17. Fruehauf J, Alberts D. In vitro drug resistance versus chemosensitivity: two sides of different coins. J Clin Oncol. 2005;23:3641-3.

18. Nagourney R. Chemotherapy sensitivity and resistance assays: a systemic review? J Clin Oncol. 2005;23:3640-1.

19. Medicare Coverage: MCAC Laboratory and Diagnostic Services Panel Human Tumor Assay Systems. Minutes of November 15-16, 1999, Meeting. $\mathrm{http}: / / \mathrm{www} . \mathrm{cms}$. hhs.gov/mcd/viewmcac.asp? where $=$ basket\&mid $=14 \&$ basket $=$ mcac: $14:$ Human + Tumor + Assay + Systems $+\% 28$ Laboratory + and+Diagnostic+Services+Panel\%29:11/15/1999. Accessed June 2006.

20. Strauss G, Herndon J, Maddaus M, et al. Adjuvant chemotherapy in stage IB non-small cell lung cancer (NSCLC): update of Cancer and Leukemia Group B (CALGB) protocol 9633. ASCO Annual Meeting Proceedings Part I. J Clin Oncol. 2006;24(18S):7007.

21. Fruehauf J. In vitro assay-assisted treatment selection for women with breast or ovarian cancer. Endocr Relat Cancer. 2002;9:171-82.

22. Doubre H, Cesari D, Mairovitz A, et al. Multidrug resistanceassociated protein (MRP1) is overexpressed in DNA aneuploid carcinomatous cells in non-small cell lung cancer (NSCLC). Int J Cancer. 2005;113:568-74

23. Volm M, Drings P, Mattern J, et al. Prognostic significance of DNA patterns and resistance-predictive tests in non-small cell lung carcinoma. Cancer. 1985;56:1396-403.

24. Gregorc V, Ludovini L, Pistola L, et al. Relevance of p53, bcl-2, and $\mathrm{Rb}$ expression on resistance to cisplatin-based chemotherapy in advanced non-small cell lung cancer. Lung Cancer. 2003;39:41-8.

25. Ludovini V, Gregorc V, Pistola L, et al. Vascular endothelial growth factor, p53, Rb, Bcl-2 expression and response to chemotherapy in advanced non-small cell lung cancer. Lung Cancer. 2004;46:77-85.

26. d'Amico T, Aloia T, Moore M-B, et al. Molecular biologic substaging of stage I lung cancer according to gender and histology. Ann Thorac Surg. 2000;69:882-6.

27. Onn A, Correa A, Gilcrease M, et al. Synchronous overexpression of epidermal growth factor and HER2-neu protein is a predictor of poor outcome in patients with stage I non-small cell lung cancer. Clin Cancer Res. 2004;10:236-43.

28. Zhang W, Stoehlmacher J, Park D, et al. Gene polymorphisms of epidermal growth factor receptor and its downstream effector, interleukin-8, predict oxaliplatin efficacy in patients with advanced colorectal cancer. Clin Colorectal Cancer. 2005;5:124-31.

29. Donnenberg V, Donnenberg A. Multiple drug resistance in cancer revisited: the cancer stem cell hypothesis. J Clin Pharmacol. 2005;45: 872-7.

30. Israeli D, Ziaei S, Gonin P, Garcia L. A proposal for the physiologic significance of mdr1 and Bcrp1/Abcg2 gene expression in normal tissue regeneration and after cancer therapy. J Theoret Biol. 2005;232: $41-5$.

31. Schiller J, Harrington D, Belani C, et al. Comparison of four chemotherapy regimens for advanced non-small-cell lung cancer. $N$ Engl J Med. 2002;346:92-8.

32. Wieand H. Chemotherapy sensitivity and response assays: are the ASCO guidelines for clinical trial design too restrictive? J Clin Oncol. 2005;23:3643-4.

33. Nevins J, Petersen R, Mukherjee S, et al. A genomic strategy to refine prognosis in early stage non-small cell lung carcinoma (NSCLC). ASCO Annual Meeting Proceedings Part I. J Clin Oncol. 2006; 24(18S):7026.

34. Leaf C. Why we're losing the war on cancer-and how to win it. Fortune. 2004 Mar 22;149(6).

\section{Discussion}

Dr David M. Jablons (San Francisco, Calif). It is a pleasure to review this paper. I have only a few questions and some observations.

What we have heard here this morning, or at least what is in the manuscript, is a retrospective analysis of a large, prospectively gath- 
ered sample of patients with early-stage resected NSCLC for the purpose of assaying in a primary cell culture system-remember, on agar-chemotherapy resistance, not sensitivity. It is an impressive experience over many years. More than 4500 fresh tumor samples were submitted to a commercial entity, Oncotech, Inc, which is a for-profit organization, for chemoresistance testing and ultimately recently gene analysis. This a reimbursable test currently that has gained some but not universal acceptance. An important distinction was brought up in the talk and needs to be reinforced: these assays are chemoresistance and not chemosensitivity, and as such the authors argue are more "credible" and more predictive of clinical response. It may be that they are right, but certainly no assessment can be made from the dataset presented today. I would agree to a certain extent that a tritium proliferation assay in a long-term culture of 5 days on agar is perhaps more quantifiable and may be more accurate. Perhaps the advantage of having a crude tumor preparation where you have a lack of tumor cell isolation and you have no macrodissection or microdissection can either be a good or a bad approach, in my opinion. It is good in the sense that it is more representative of the actual tumor perhaps in its milieu with stromal cells and vascular cells and immune cells and maybe the purported cancer stem cells. Yet it may be that the lack of tumor cell isolation allows or accounts for why this high chemoresistance is observed. Remember that these cells are being cultured, as I understood from the paper, in very high supraphysiologic concentrations of chemotoxic agents, 10-fold to 100-fold higher, which are at least 2 to $3 \log$ orders higher than they would ever see in the microenvironment in vivo. So one quick question that comes to mind is, did these resistance patterns vary at all with histology? For instance, there is no comment in the paper that there was an association with adenocarcinoma versus squamous, subsets of adenocarcinomas that we know are notoriously more chemoresistant-for example, neuroendocrine, large cell, bronchoalveolar. Was there any attempt at Oncotech or otherwise to classify that? Also, in the molecular analysis, was there any microdissection of the tumors, because without that there is no way to safely contend or conclude that what you are measuring is really tumor focus. Finally, what was the FedEx factor in this? We have all done these experiments and sent our samples, and I am curious there is no comment as to the overall success rate. Having been in the gene vaccine business for a long time, we were surprised that we could get $80 \%$ viability shipping tumor samples for fresh autologous vaccines across country, so how was it in this? Finally, how realistic is this assay? The answer, in my opinion, is not very. Despite the many objections and the artifactual-ness of the assay, it has been shown as represented in the paper in several other tumor systems, ovarian and breast cancer, to have some prognostic activity and validity. So I would ask the authors, who are busy clinicians and have a large thoracic surgery and lung cancer practice-this has not been validated prospectively and needs to be, I agree — what have you been doing either off study or on study in small pilot studies in your institution to validate this? Do you use this as a proponent of this therapy, because it is highly controversial in this assay? Do you personally use it to guide therapy? If not, why not?

In the interest of time, I will limit the comments regarding stage $1 \mathrm{~B}$ adjuvant chemotherapy. I think it can be safely concluded now, and Eric is in the back and can concur, at ASCO just a few weeks ago the analysis of the CALGB data is now a negative trial. The larger trials and more robust longer follow-up trials, ANITA, IALT, and others, showed no benefit for adjuvant platinum-based chemotherapy in patients with stage 1B disease that are node negative. So I no longer believe that is standard of care. There are many issues with the molecular analysis of p53. It is only one gene. It is notoriously inaccurate by immunohistochemistry. And p14, MDM2, there are lots of way to skin that gene, and when you just look for immunohistochemistry, it is very unpredictable and unreliable.

In light of the impending revolution in molecular classification and fingerprinting of tumors and emergence of novel targeted therapies and agents, just keep in mind that there are nearly 300 new agents, not to mention Irv [Dr Irving Weismann]'s 300-odd companies I'm sure, that are coming into clinical testing in the next 3 to 5 years. Is there a role in this day and age for the "old world" chemotherapy and a reason to believe that in vitro chemoresistance testing can be useful to select efficacious agents? Molecular analysis is sexy and exciting and is, remember, mostly prognostic at this point, but not therapeutic. So in the next 3 to 5 years, perhaps longer, chemotoxic, cytotoxic chemotherapy is here to stay. As such, anything, if validated prospectively, that can direct unselected empiric therapy to select patient populations and their tumors would be a meaningful advance. As the saying goes, in the land of the blind, the one eye is king. The time is ripe to test this and other in vitro chemoresistance assays for clinical response and survival as their end points. This is yet to be done in NSCLC.

It is time to put the assay to the real "test" road and design an adjuvant trial for the intergroup that has drug selection based on some form of rational in vitro testing as a basis, and only then can we determine whether this is a valid approach.

Dr d'Amato. Thank you, Dr Jablons. I'll try to address as many of your questions as I can.

Currently, it is difficult to get many of the medical oncologists to treat patients based on this assay. We do have several at the University of Pittsburgh who will, and when I was in practice in Southern California, I had several who would treat people based on the assay. I put forward a proposal to the CALGB last year to use the assay as a validation trial, as a single arm, nontreatment trial that would serve to validate the assay. The problem with an assay-directed versus empiric trial obviously is that that would create a subset of patients in whom you would eliminate platinum. Because that has been the standard of care for NSCLC, eliminating a platinum agent if a patient were resistant would be beyond that, so a clinical validation, at least for the resectable disease, is important. Those proposals are being put forth to the intergroup.

Regarding the assay and the mechanics of the assay, somewhere between $75 \%$ and $85 \%$, what we call a valuability, meaning viable tumor cells that can be cultured, arrived by FedEx when the tumors were prepared properly and sent and did not sit on a loading dock. There are extremes from that, I'm sure, as you have seen in your own experience with the vaccine work.

Stromal cells and vascular endothelial cells are inhibited in the assay conditions on agarose and this has been well established for almost 20 years, when Hamburg and Solomon initially learned 
how to culture tumor cells in the older (clonagenic) assays, so I don't believe that right now is a particular issue.

There are some differences between the frequencies of in vitro resistance to chemotherapeutic agents in non-small cell tumor cultures of different histology. For example, as we may expect from clinical observations, NSCLC tumors having bronchoalveolar histology appear to exhibit more in vitro resistance to most agents. These data are currently being analyzed and have yet to be validated.
Regarding micro dissection, this is not performed for standard immunohistochemistry.

I believe that the molecular markers that we have chosen are difficult to assess in terms of the resistance assay. I also agree that the immunohistochemistry as end point measurements are not as accurate, particularly with EGFR. The missing link here with these markers is the clinical data. Finding associations is interesting, but are they meaningful? As you have noted, in the manuscript they probably are not meaningful in this context without clinical validation. 